\title{
GCU
}

Glasgow Caledonian

University

University for the Common Good

\section{Simple finite element models for use in the design of therapeutic footwear}

Spirka, Thomas A.; Erdemir, Ahmet; Spaulding, Susan Ewers ; Yamane, Ann; Telfer, Scott; Cavanagh, Peter R.

Published in:

Journal of Biomechanics

DOI:

10.1016/j.jbiomech.2014.07.020

Publication date:

2014

Document Version

Author accepted manuscript

Link to publication in ResearchOnline

Citation for published version (Harvard):

Spirka, TA, Erdemir, A, Spaulding, SE, Yamane, A, Telfer, S \& Cavanagh, PR 2014, 'Simple finite element models for use in the design of therapeutic footwear', Journal of Biomechanics, vol. 47, no. 12, pp. 2948-2955. https://doi.org/10.1016/j.jbiomech.2014.07.020

\section{General rights}

Copyright and moral rights for the publications made accessible in the public portal are retained by the authors and/or other copyright owners and it is a condition of accessing publications that users recognise and abide by the legal requirements associated with these rights.

Take down policy

If you believe that this document breaches copyright please view our takedown policy at https://edshare.gcu.ac.uk/id/eprint/5179 for details of how to contact us. 
2

3 4

5

6

7

\title{
SIMPLE FINITE ELEMENT MODELS FOR USE IN THE DESIGN OF THERAPEUTIC FOOTWEAR
}

\author{
${ }^{1}$ Thomas A. Spirka, ${ }^{2}$ Ahmet Erdemir, ${ }^{1}$ Susan Ewers Spaulding, ${ }^{1}$ Ann Yamane, ${ }^{1,3}$ Scott Telfer and \\ ${ }^{1}$ Peter Cavanagh
}

${ }^{1}$ Department of Orthopaedics and Sports Medicine, University of Washington, Seattle, WA, USA

${ }^{2}$ Computational Biomodeling (CoBi) Core and Department of Biomedical Engineering, Lerner Research Institute, Cleveland Clinic, Cleveland, OH, USA

${ }^{3}$ Institute of Applied Health Research, Glasgow Caledonian University, UK

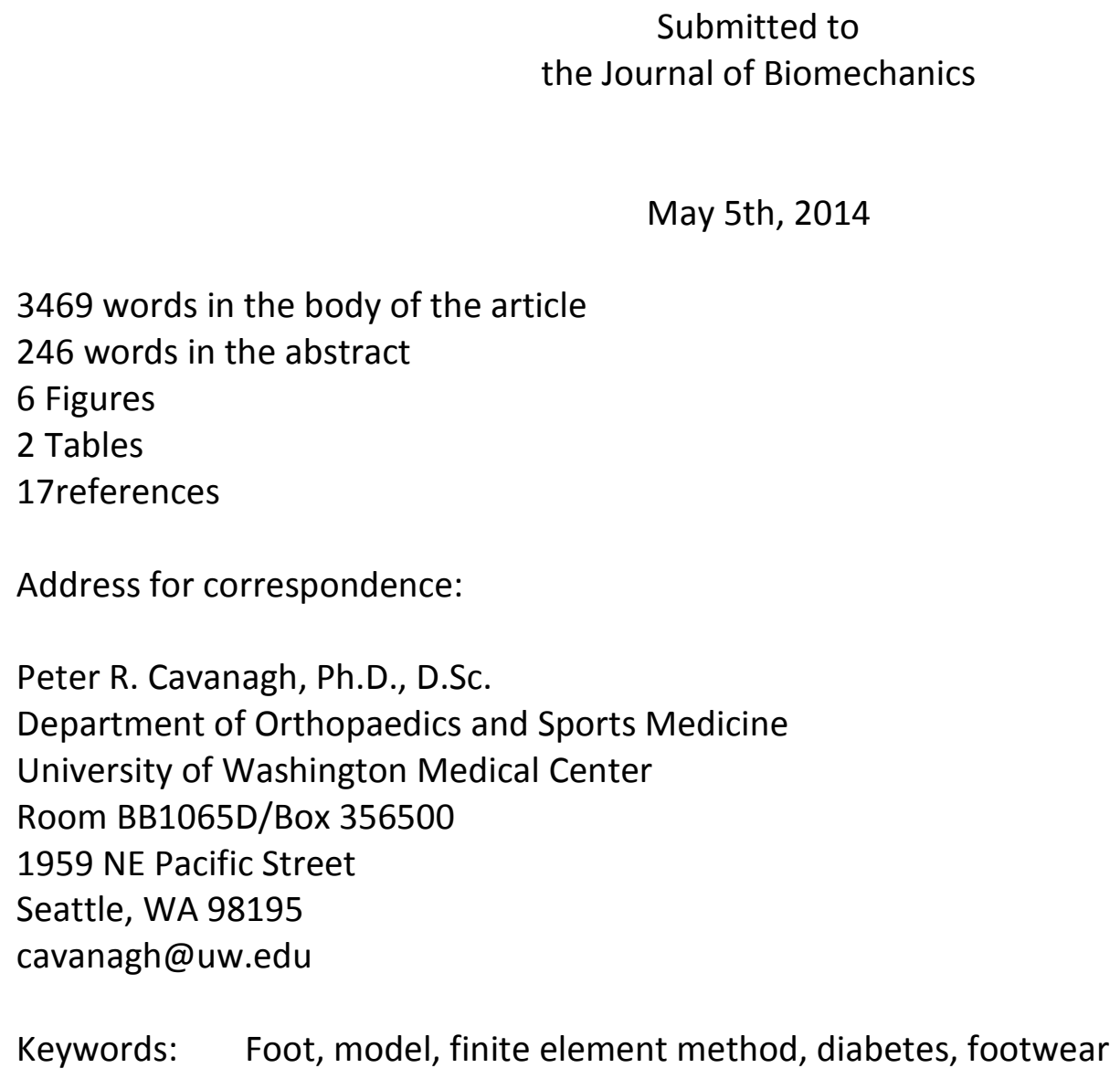


(246/250 words)

\section{Abstract}

Therapeutic footwear is frequently prescribed in the cases of rheumatoid arthritis and diabetes to relieve or redistribute high plantar pressures in the region of the metatarsal heads. Few guidelines exist as to how these interventions should be designed and what effect such interventions actually have on the plantar pressure distribution. Finite element analysis has the potential to assist in the design process by either refining a given intervention or identifying an optimal intervention without having to actually build and test each condition. However, complete and detailed foot models based on medical image segmentation have proven time consuming to build and computationally expensive to solve, hindering their utility in practice. Therefore, the goal of the current work was to determine if a simplified patient-specific model could be used to assist in the design of foot orthoses to reduce the plantar pressure in the metatarsal head region. The approach is illustrated by a case study of a diabetic patient experiencing high pressures and pain over the fifth metatarsal head. The simple foot model was initially calibrated by adjusting the individual loads on the metatarsals to approximate measured peak plantar pressure distributions in the barefoot condition to within $3 \%$. This loading was used in various shod conditions to identify an effective orthosis. While model predictions were generally greater than peak pressure measured for the shod conditions (by approximately 16\%), the modeling approach enabled virtual prototyping of the orthoses, identifying the most favorable approach to redistribute the patient's plantar pressures. 


\section{Introduction}

Therapeutic footwear is often prescribed to redistribute plantar pressures and attenuate peak pressures in at-risk regions of the foot both to relieve pain in the case of patients with rheumatoid arthritis and to reduce the risk of plantar ulcers in patients with diabetes (Hennessy et al. 2012, Boulton et al. 2004). However, very few guidelines exist as to how these interventions should be designed and what effect such interventions actually have on the plantar pressure distribution (Waaijman et al. 2012). In addition, how footwear designs can be individualized to accommodate patient-specific foot anatomy is not clear. Frequently, the design of an intervention is based on the clinical experience of the pedorthist.

Finite element analysis has the potential to assist in the design process by allowing the clinician to evaluate the effectiveness of many different interventions in order to identify which solution could provide the greatest benefit to a given patient, without having to actually build and test each modification. Several authors have proposed the construction of detailed foot models that incorporate as many of the structures of the foot as possible (Franciosa et al. 2012, Qiu et al. 2011, Hsu et al. 2008, Cheung, \& Zhang 2008, Actis et al. 2008) for the purpose of designing footwear. However, development of a full-foot patient-specific model can be a laborintensive and time consuming process which frequently results in a computationally expensive model. This limits the feasibility of utilizing models in a translational sense, for a large number of patients and for the often iterative process of footwear design. Simplified two-dimensional models of the foot with somewhat limited loading conditions have been proposed by Yarnitzky et al. (2006). The goal of the current work was to determine if a simplified 3D model 
customized for gross patient-specific anatomy could be used to assist in the design of footwear interventions to reduce plantar pressures at targeted forefoot regions. The approach is illustrated by a case study of a diabetic patient experiencing high pressures and pain under the fifth metatarsal head and elevated pressure under the first metatarsal head.

\section{Methods}

Finite element representations of the metatarsal region of the foot

Computed tomography (CT) of the left foot were acquired with approval of the University of Washington Institutional Review Board from a 46 year-old male (height $=1.8 \mathrm{~m}$, weight $=74 \mathrm{~kg}$ ) with Type I diabetes. He presented with callus under the $1^{\text {st }}$ and $5^{\text {th }}$ metatarsal heads, pain under the 5 th despite dense peripheral neuropathy, and a plantarflexed $1^{\text {st }}$ ray. Images were acquired on a GE Lightspeed VCT (GE Medical Systems, Milwaukee, WI) with a slice thickness $0.625 \mathrm{~mm}$. Image resolution was 512 pixels $\times 512$ pixels with pixel size of $0.6 \mathrm{~mm}$ x $0.6 \mathrm{~mm}$. The image processing software Scan IP (Simpleware Ltd, Exeter, UK) was used to acquire the measurements listed in Table 1. Briefly, the minimum tissue thickness beneath each metatarsal head (MTH), the length of each metatarsal bone, the maximum width of each $\mathrm{MTH}$, the distance between the center point of each $\mathrm{MTH}$ relative to the neighboring MTH and the angle the metatarsal bone makes with a straight line running tangent to the plantar surface were all measured. The accuracy and reliability of the above measurements is observerdependent: for example, five repeat measurements of tissue thickness under MTH2 and the 
width of MTH2 made by two observers resulted in mean values that differed by less than 0.1 $\mathrm{mm}$ and $0.5 \mathrm{~mm}$ respectively, but the coefficients of variation for the two observers were $1 \% / 3 \%$ and $5 \% / 11 \%$ (observers 1 and 2 , thickness/width).

Measurements of gross metatarsal anatomy and tissue thickness were incorporated into a finite element model of the metatarsal region of the foot that utilized simple shapes to represent the bones and the surrounding tissue utilizing Abaqus CAE Version 6.10 (Simulia, Providence, RI). The metatarsal bones were modeled as rigid structures using a combination of a cylinder and a sphere (Figure 1a). The diameter of the sphere representing the metatarsal head was based on the maximum width of each metatarsal head measured using the CT scans. The overall length of the metatarsal was determined from measurements acquired from the CT scans while the shaft of the bones were assumed to be cylindrical with a diameter equal to $80 \%$ of the metatarsal head diameter. The metatarsal bones were then oriented relative to the center point of the third metatarsal head. Finally, the bones were rotated about an axis running perpendicular to the long axis of the bone and through the center point of the spherical metatarsal head to match the angle of the bones observed in the CT scans when the plantar aspect of the foot was parallel to the ground plane. The resulting bone positions can be seen in Figure $1 \mathrm{~b}$. Observation of the patient's barefoot plantar pressure, which showed that peak MTH pressure occurred very soon after heel raise, confirmed this approximation for the MT angles of this model.

Ligaments were incorporated into the model to accommodate restraints on mediolateral splay of the metatarsals. Tension-only truss elements, having elastic material 
119 properties $(E=260 \mathrm{MPa}$, Poisson's Ratio $=0.3)$ with uniform cross sectional area of $\left(18.4 \mathrm{~mm}^{2}\right)$,

120

121

122

were used for this purpose (Cheung, \& Zhang 2008). A total of 11 ligament locations were based on the descriptions and drawings provided in Primal Pictures 3D Anatomy Software (Primal Pictures Limited, London, UK). Plantar metatarsal ligaments were attached between adjacent spheres representing each $\mathrm{MTH}$, with attachment points positioned on the plantar surface of the sphere approximately $3 \mathrm{~mm}$ medially or laterally from the center axis when viewed in the coronal plane. In a similar manner, plantar and dorsal inter-metatarsal ligaments were attached between adjacent bones approximately $5 \mathrm{~mm}$ from the proximal end of the cylinder representing each metatarsal shaft. The connections of the metatarsal bones to the cuneiforms and the cuboid, were modeled using universal joint connector elements (Abaqus $6.10,2010)$ to allow for the plantar/dorsiflexion and splay of the metatarsals. The cuneiforms and cuboid were not modeled explicitly but abstracted as a rigid body.

A tissue block (Figure 1c) was modeled as a nearly incompressible Ogden hyperelastic material ( $\mu=0.01645, \mathrm{MPa}, \alpha=6.82$, effective Poisson's Ratio $=0.49$, (Erdemir et al. 2006). The block was defined such that the plantar surface of the bones were embedded within the block. To accommodate the presence of the metatarsal bones within the soft tissue, Boolean subtraction was used to remove a volume in the shape of metatarsals from the tissue block. Tie constraints between the metatarsal bone surfaces and the surrounding tissue were defined. The arch of the foot was assumed to have an ellipsoidal shape that was defined by the navicular height $(13 \mathrm{~mm})$ together with anterior-posterior $(152 \mathrm{~mm})$ and medial-lateral measurements (75mm) of the arch taken from the CT images. This ellipsoidal shape was then Boolean- 
140 subtracted from the tissue block to define the arch of the foot. The final resulting model of the

141 metatarsal region of the foot can be seen in Figure 1d. The soft tissue and bones were meshed 142 using standard linear tetrahedral elements (C3D4) with a characteristic length of $1.8 \mathrm{~mm}$ 143 (Cheung \& Zhang, 2008; Isvilanonda et al., 2011). A convergence analysis showed that reducing 144 the characteristic element length by $50 \%$ resulted in $<5 \%$ changes in the predicted contact 145 pressures. In addition, a preliminary analysis using nearly incompressible tissue properties (as 146 stated above) illustrated that the predictive capacity for internal and external tissue mechanics 147 metrics were similar to that of tetrahedral element with hybrid formulation (C3D4H), (the 148 average difference in contact pressures was $\sim 7 \mathrm{kPa}$ ). Previously, Tadepalli et al. (2011) have 149 shown the adequacy of tetrahedral elements with hybrid formulation for simulation of nearly 150 incompressible tissue behavior. The element selection used in the present study provided significant cost savings in computational time ( 2.5 times faster). The bones were modeled as 152 rigid elements to save computational cost.

As the structures distal to the MTHs were not modeled, the margins of the soft tissue universal joint element defined for each metatarsal. This resulted in constrained translations and rotations at one end of the tarsometatarsal joints where the rigid body movements of the metatarsals were defined by the universal joint element. The initial model was designed to 159 simulate the barefoot condition by placing the plantar surface of the model in contact with a 
rigid structure representing the floor that was fully constrained against translation or rotation. Contact at the foot-floor interface was defined by a static coefficient of friction of 0.5 .

The model was loaded by applying a concentrated force load to the node closest to the plantar surface of each metatarsal head. To determine the magnitude of each load, the patient walked barefoot at a self-selected speed using a first step protocol across an EMED pedography platform (Novel GMBH Munich Germany). The amount of force exerted under the metatarsal heads at the time point corresponding to peak pressure in the gait cycle was determined by integrating pressure over the region of interest. The loads were then modified by inspection based on the resulting predicted plantar pressures until the predicted maximum pressure under each metatarsal head matched the maximum measured pressures in the corresponding region as closely as possible. Once determined, this loading was also utilized for simulations of footwear interventions as described below. Peak regional pressures were obtained from the model output by identifying the largest pressures in a circular region centered on the MTH that did not interfere with adjacent regions.

\section{Footwear Interventions}

Prior to the modeling effort, an experienced certified prosthetist/orthotist (CPO) worked with the subject to design personalized footwear that would reduce the plantar pressure at locations determined by the plantar pressure system. In-shoe plantar pressure data was gathered as the participant walked at a self-selected speed over level ground in his shoes and in 
181 the various interventions used. The CPO did not have access to model results but was

182 unconstrained in her use of materials or geometries.

To determine the ability of the model to predict the effect of footwear interventions on peak plantar pressures under the metatarsal heads, two types of common footwear interventions designed to reduce plantar pressures in this region were modeled: 1) flat insoles simulating materials of different hardness; and 2) metatarsal pads to relieve focal pressure by increasing pressure at adjacent pressure-tolerant areas. Unless stated, footwear components were modeled as rectangular blocks $110 \mathrm{~mm}$ wide by $100 \mathrm{~mm}$ long using Abaqus C3D8 hexahedral elements.

The shoe midsole and outsole were modeled as a $13 \mathrm{~mm}$ thick flat block (firm crepe) meshed with hexahedral elements with a characteristic length of $4.3 \mathrm{~mm}$. Flat insoles of uniform thickness were meshed on top of the midsole with hexahedral elements with a characteristic length of $2 \mathrm{~mm}$. A convergence analysis was performed for each footwear component, with meshes deemed to be acceptable when reducing the element length by $50 \%$ resulted in changes in contact pressures and von Mises stresses of $<5 \%$. Three different insole materials, Microcell Puff ${ }^{\circledR}\left(9.5 \mathrm{~mm}\right.$ thick), Poron Cushioning ${ }^{\circledR}\left(6 \mathrm{~mm}\right.$ thick) and Medium Plastazote ${ }^{\circledR}(6 \mathrm{~mm}$ thick) were modeled with the hyperfoam material properties shown in Table 2 (Petre et al. 2006). The midsole was constrained by tying the bottom surface to the top surface of the floor, while the bottom surface of the insole was tied to the top surface of the midsole, resulting in no shear movement between the materials. Contact between the plantar tissue and the insole was defined by a static coefficient of friction of 0.5 . 
To study the effects of metatarsal pads (MPs) on the plantar pressure, a metatarsal pad $64 \mathrm{~mm}$ long, $54 \mathrm{~mm}$ wide, and $9.5 \mathrm{~mm}$ at the highest point with $9.5 \mathrm{~mm}$ fillet around its top edge (Figure 2a) was given the properties of Microcell Puff ${ }^{\circledR}$ and placed on top of a $6 \mathrm{~mm}$ thick medium Plastazote ${ }^{\circledR}$ insole in 3 different orientations (Figure $2 b, 2 c$ and $2 d$ ). The MP placements were: a) $6.5 \mathrm{~mm}$ proximal to the center point of MTH3; b) directly under the center point of $\mathrm{MTH} 3$; c) $10 \mathrm{~mm}$ lateral to $\mathrm{MTH} 3$ to simulate an improper placement. In these simulations, the bottom surface of the pad was tied to the top surface of the insole.

The plantar pressure data from most of the models were validated using pressure distributions measured directly from the subject with an EMED platform for barefoot walking and a Pedar in-shoe system (Novel GMBH, Munich, Germany) for the footwear conditions. The different positions of the MP ( $b$ and $c$ above) were not measured directly because: 1 ) the inshoe sensors did not have sufficient resolution to make a meaningful measurement; 2) considerations of patient safety suggested that only reasonable modifications should be attempted.

\section{Results}

By modifying the proportion of the total measured load applied to the each metatarsal head in the model, a load condition was identified in which predicted barefoot MTH plantar pressures from the model were within $25 \mathrm{kPa}$ of measured values (3\%). The final loads applied to each metatarsal head were $304 \mathrm{~N}, 115 \mathrm{~N}, 5 \mathrm{~N}, 30 \mathrm{~N}, 74 \mathrm{~N}$ on metatarsal heads $1-5$ respectively. 
223 These loads, which resulted in differences of $20 \mathrm{kPa}, 7 \mathrm{kPa}, 21 \mathrm{kPa}, 1 \mathrm{kPa}$, and $6 \mathrm{kPa}$ between the measured and predicted peak pressures for each of the metatarsal heads, were then applied to 225 all footwear conditions. Comparisons between predicted results and measured values can be seen for: a) the barefoot condition in Figure 3; b) 3 different insoles in Figure 4; and c) MPs in various locations in Figure 5. The overall patterns of measured and predicted plantar pressures are most similar in the flat insole conditions although the peak pressures are consistently over-predicted at the MTHs by the simulation. The predicted pattern in the case of the MT pad shows similar elevated peak pressure anterior to the MTHs but the model results in a more detailed distribution than is available from the large elements in the Pedar insole (see discussion). at 14.2 minutes (wallclock time) and the MP model the slowest at 90 minutes. 


\section{Discussion}

constructing a model to inform footwear design difficult. This study was intended to test the

to characterize key aspects of the patient's anatomy that may affect plantar pressure (Morag et

al. 1997) while allowing for multiple iterations of different footwear interventions. Currently, the most common approach is the construction a FE model of the entire foot based on 
The fact that the model estimates of peak contact pressures are larger than the

measured values is not surprising since the spatial resolution of the in-shoe measurement system is only $\sim 10 \mathrm{~mm} \times 10 \mathrm{~mm}$ while the individual surface faces of the elements in the model are triangles with initial side lengths of $1.8 \mathrm{~mm}$. Differences in actual vs. modeled soft tissue properties may also have contributed to the difference. The model is also able to detect local areas of stress concentration (e.g. those associated with the MP in Figure $5 d$ ). This is a frequent clinical concern and a topic that deserves further investigation through simulation since it cannot readily be measured using low-resolution in-shoe sensors.

There are a number of limitations to the present approach. Currently CT or MRI images are needed to establish the model parameters. Further work needs to be done to determine if these values could be obtained using ultrasound, which will enable routine measurements to be made in a clinical setting. The model presented here incorporates several assumptions that are typical of larger models: the soft tissue is modeled as a single lumped tissue layer and represented by a single hyper-elastic material model that is not patient specific. We examined the sensitivity of the plantar pressure results to changes in tissue mechanical properties. A variation of $+/-25 \%$ in ligament properties results only in a $\sim 3 \%$ change in peak plantar pressure. Plantar tissue properties were, however, more influential: a variation of $+/-1$ sd in the tissue properties as reported by Erdemir et al (2006). resulted in a 33\% change in the predicted plantar pressure. This large effect emphasizes the importance of personalized measures of plantar tissue properties in future models. While the incorporation of differentiated tissue layers to allow internal stresses to be accurately predicted (Petre et al. 2013) and viscoelasticity 
may be feasible, such refinements would significantly increase the labor cost during model development. Viscoelasticity, if modeled, will also increase the computational expense and such adjustments to model fidelity are only needed if research and/or clinical questions dictate their use. The hyperelastic definition used in this model can be justified since the focus is on the response of the tissue for different footwear conditions potentially loaded at a similar rate.

A critical assumption in this model is that the force loading of the metatarsal heads is the same during both barefoot and shod gait - implying that the gait pattern does not change as a result of footwear or the interventions. While this is indeed a limitation, it also established the power of the approach to conduct a predictive analysis of an insole. It is well known that footwear affects the plantar pressures at the foot-shoe interface. However, the relative contributions of footwear and changes in the gait pattern are not known. A further limitation of this model and the full-foot models published to date is that only a single instant in time of the gait cycle is considered in the quasi static models, in our case the instant of peak plantar pressure. In reality, the gait cycle is a dynamic event in which the pressures generated and the loads responsible for generating these pressure change continuously. However, the complexity of the foot and the need to reposition structures and alter loads as time evolves hinders development and utility of dynamic models of the foot. Future modeling efforts should also include a more accurate representation of the tarso-metatarsal articulations and their influence on metatarsal orientation.

A review of the results of the simulations (Figure 6) indicates that the use of a metatarsal pad positioned such that the anterior margin of the pad was proximal to MTH3 
resulted in the greatest unloading effect at both major regions of interest (MTH1 and MTH5). Although the absolute magnitude of the peak pressure differed between the model and experimentally measured values, the ranking of unloading predicted by different simulations may still be useful in directing clinical attention to the best intervention.

\section{Conclusions}

footwear design. Further exploration of the model with more complex footwear interventions and a diverse group of foot types is now warranted. In addition to patient-specific use, the results from a validated model may also find a more general use as a teaching tool for pedorthists to provide insight into the quantitative and qualitative effects of footwear modifications that are typically constructed.

\section{Acknowledgments}

This work was supported by This study was supported by NIH Grant 7R01HD037433-08 (PI: Cavanagh). Scott Telfer is funded through the People Programme (Marie Curie Actions) of the European Union's Seventh Framework Programme (FP7 2007-2013) under REA Grant Agreement No. PIOF-GA-2012-329133. 
326 Conflict of Interest

327 Peter Cavanagh has equity in DIApedia LLC and Ahmet Erdemir has equity in innodof LLC

328 
329 Table I: Measurements acquired from CT scan for construction of the simplified model

\begin{tabular}{|c|c|c|c|c|c|}
\hline & $\begin{array}{c}\text { 1st } \\
\text { Metatarsal }\end{array}$ & $\begin{array}{c}2^{\text {nd }} \\
\text { Metatarsal }\end{array}$ & $\begin{array}{c}3^{\text {rd }} \\
\text { Metatarsal }\end{array}$ & $\begin{array}{c}4^{\text {th }} \\
\text { Metatarsal }\end{array}$ & $\begin{array}{c}5^{\text {th }} \\
\text { Metatarsal }\end{array}$ \\
\hline Width of MTH (mm) & 25 & 15 & 13 & 13 & 13 \\
\hline $\begin{array}{l}\text { Medial(+)/lateral(-) } \\
\text { distance relative to } \\
\text { MTH03 }(\mathrm{mm})\end{array}$ & 41 & 17 & 0 & -15 & -35 \\
\hline $\begin{array}{l}\text { Anterior(+)/posterior(-) } \\
\text { distance relative to } \\
\text { MTH03 }(\mathrm{mm})\end{array}$ & 0 & 0 & 0 & -10 & -23 \\
\hline $\begin{array}{l}\text { Thickness of tissue } \\
\text { under MTH (mm) }\end{array}$ & $8^{*}$ & 14 & 10.5 & 8.5 & 6.5 \\
\hline $\begin{array}{l}\text { Angle relative to } \\
\text { plantar surface }\left({ }^{\circ}\right)\end{array}$ & 30 & 29 & 25 & 23 & 22 \\
\hline Length of MTH (mm) & 64 & 73 & 74 & 77 & 74 \\
\hline
\end{tabular}

* Average thickness underneath the lateral and medial sesamoids 
332 Table 2: Material Properties of Insole Materials (Petre MT, 2006)

333

\begin{tabular}{|l|c|c|c|c|}
\hline & $\mu_{1}$ & $\alpha_{1}$ & $\beta_{1}$ & Thickness \\
& (Mpa) & & & $(\mathbf{m m})$ \\
\hline Microcell Puff ${ }^{\circledR}$ & 1.34 & 28.07 & 0.06 & 9.5 \\
\hline Poron Cushioning ${ }^{\circledR}$ & 0.620 & 34.46 & 0.04 & 6.0 \\
\hline Plastazote ${ }^{\circledR}$ Medium & 0.488 & 26.09 & 0.07 & 6.0 \\
\hline Firm Crepe & & & & \\
\hline
\end{tabular}


Figure Legends:

Figure 1: Model design and construction: a) Simplified shapes used to define the metatarsal bones. b) Bone orientation showing relative position and angle relative to the plantar surface. c) Geometry of the soft tissue block. d) Final model illustrating the barefoot condition

Figure 2: Modeling of a metatarsal pad: a) Geometry of the metatarsal pad $(6.4 \mathrm{~cm}$ long, $5.4 \mathrm{~cm}$ wide, $0.95 \mathrm{~cm}$ at the highest point). b) Location of the metatarsal pad posterior to the metatarsal heads. c) Metatarsal pad centered under the $3^{\text {rd }}$ metatarsal head. d) Improper placement of the metatarsal pad with the pad shifted medially $10 \mathrm{~mm}$ from the position shown in (c).

Figure 3: Comparison of measured (a) and predicted (b) plantar pressure for the barefoot condition. Measured and predicted peak pressure values were $925 \mathrm{kPa}$ and $900 \mathrm{kPa}$ respectively.

Figure 4: Comparison of measured (left hand side) and predicted (right hand side) plantar pressure when insoles composed of Microcell Puff ${ }^{\circledR}$ (Top), Poron Cushioning ${ }^{\circledast}$ (middle), and Plastazote Medium ${ }^{\circledast}$ (bottom) are incorporated into the model. All three insole materials were successful in reducing the plantar pressure in the region when compared to the barefoot pressure with the Poron Cushioning ${ }^{\circledR}$ insole showing the greatest effect on plantar pressure (both in silico and in vivo).

Figure 5: Effect of incorporating a metatarsal pad on the predicted and measured plantar pressure. (a) Measured plantar pressure when a metatarsal pad was incorporated into the footwear. (b) Metatarsal pad located posterior to the metatarsal heads. (c) Metatarsal pad centered under the $3^{\text {rd }}$ metatarsal head. (d) Metatarsal pad shifted medially $10 \mathrm{~mm}$.

Figure 6: Predicted change in peak pressure (in $\mathrm{kPa}$ ) at each $\mathrm{MTH}$ for all interventions compared to the standard shod condition. 
$360 \quad$ Figure 1

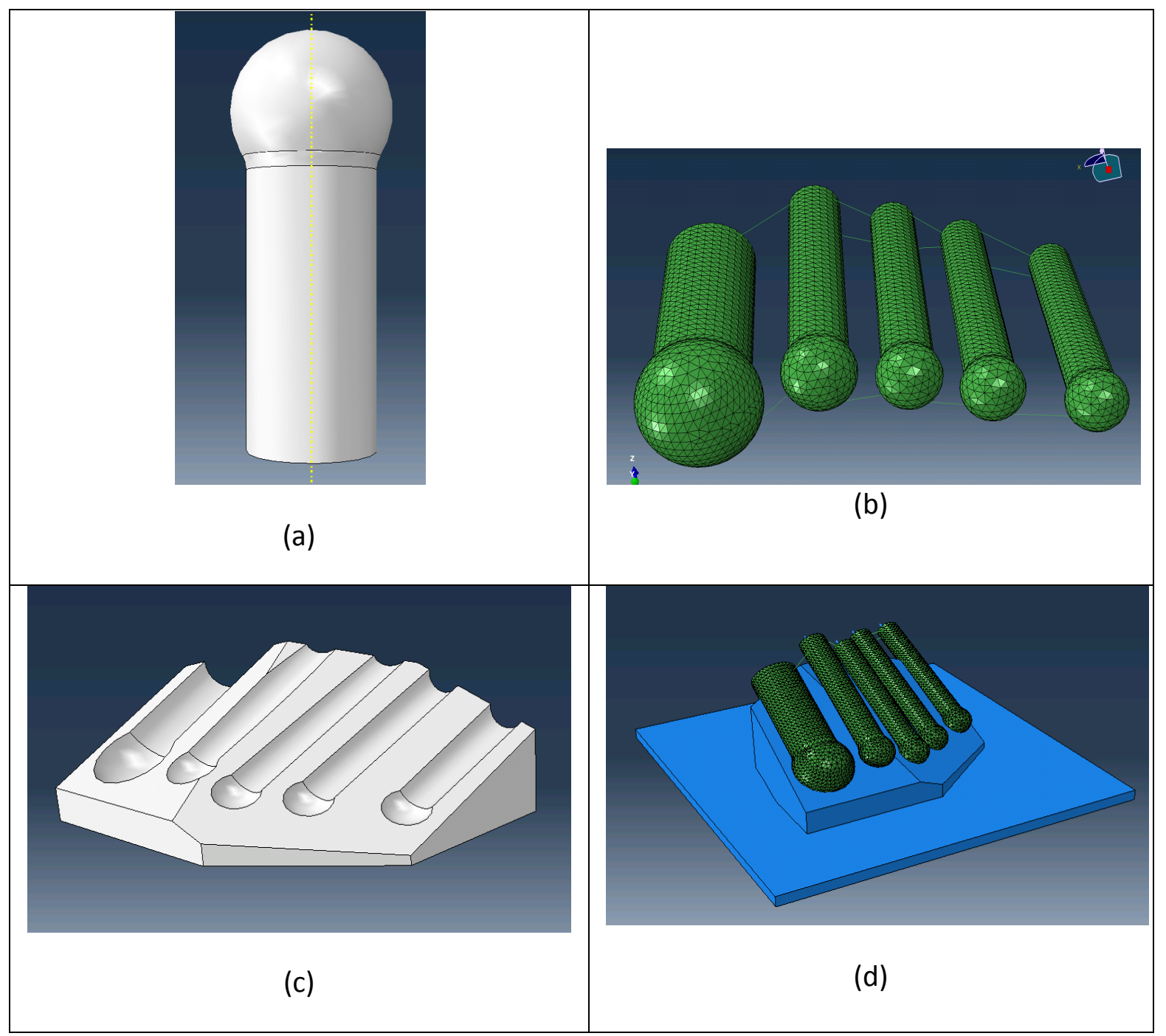

361 
Figure 2

363

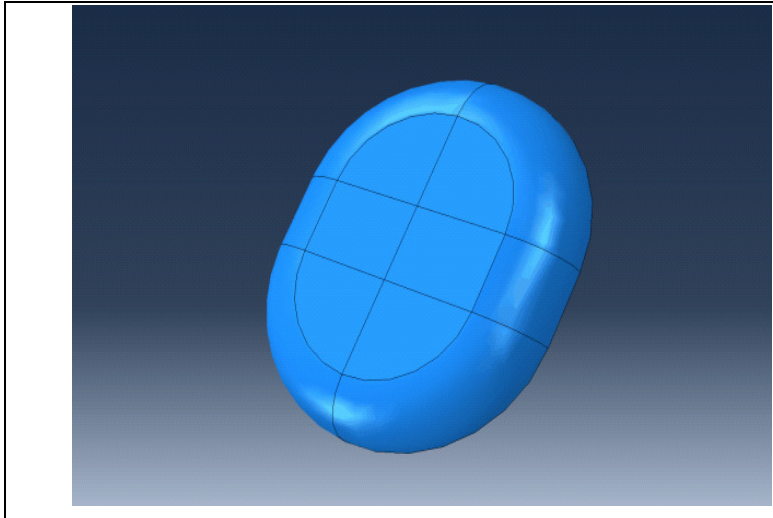

(a)

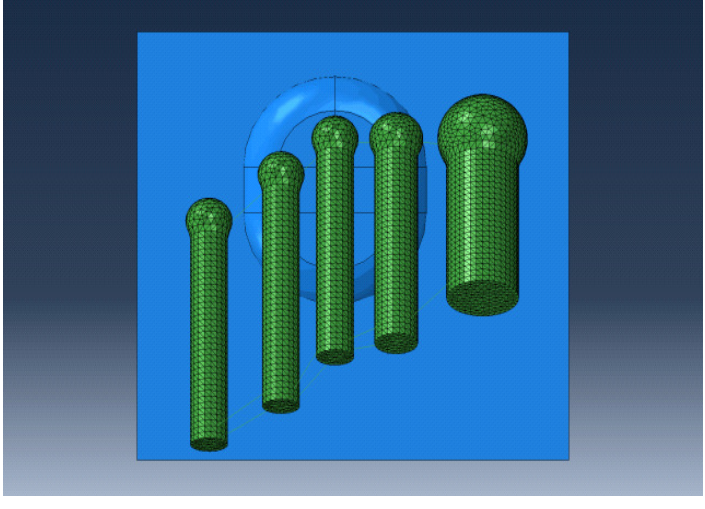

(c)

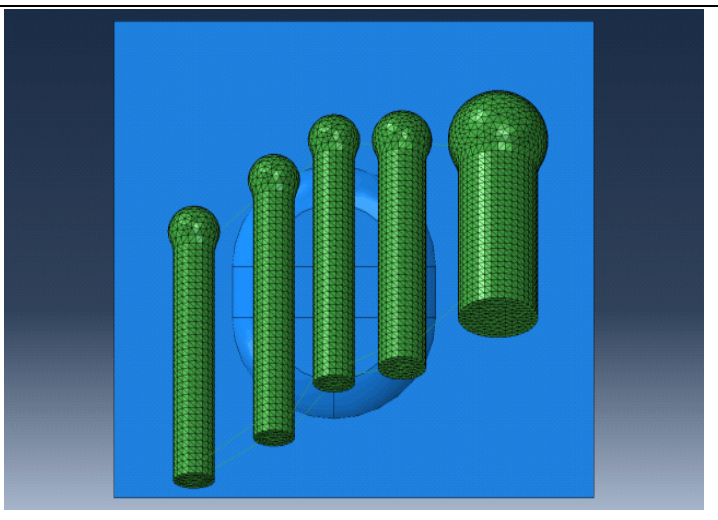

(b)

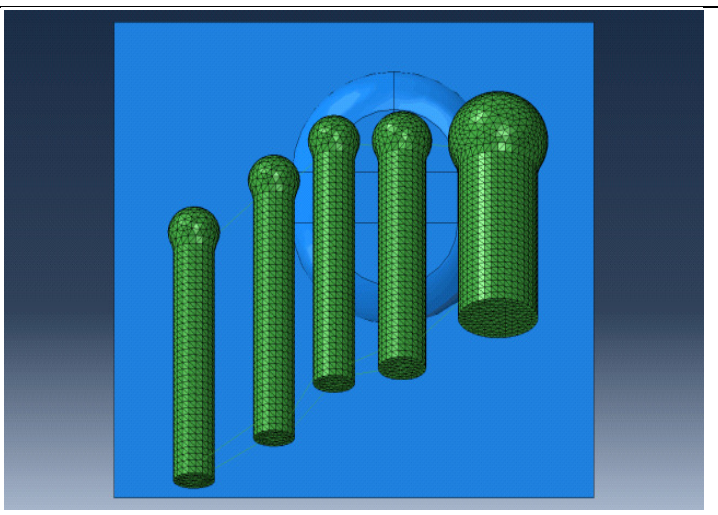

(d)

364 
369 Figure 3

370

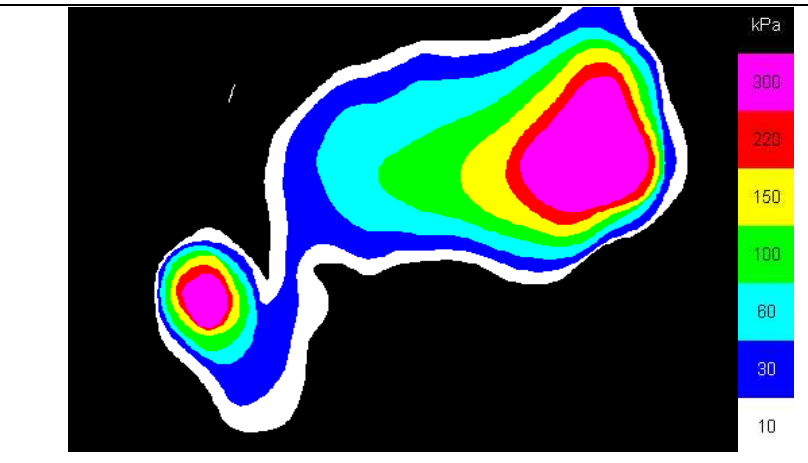

(a)

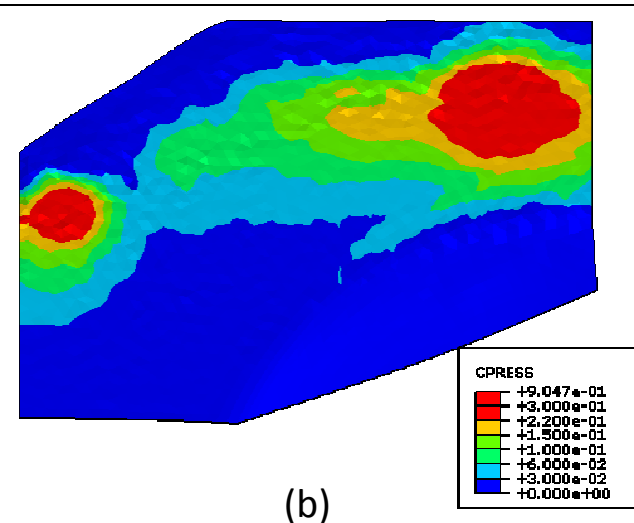

(b) 


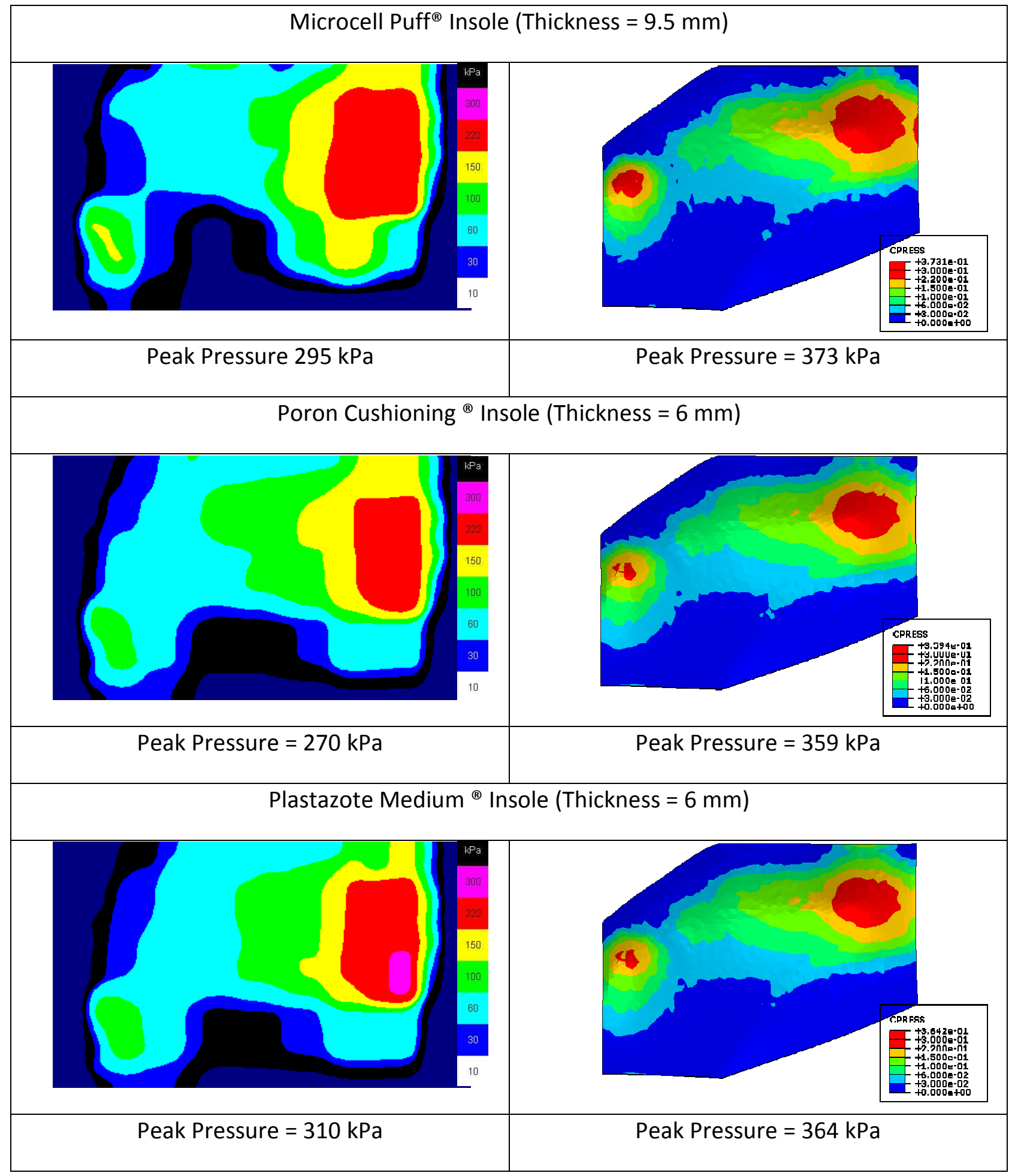


Figure 5

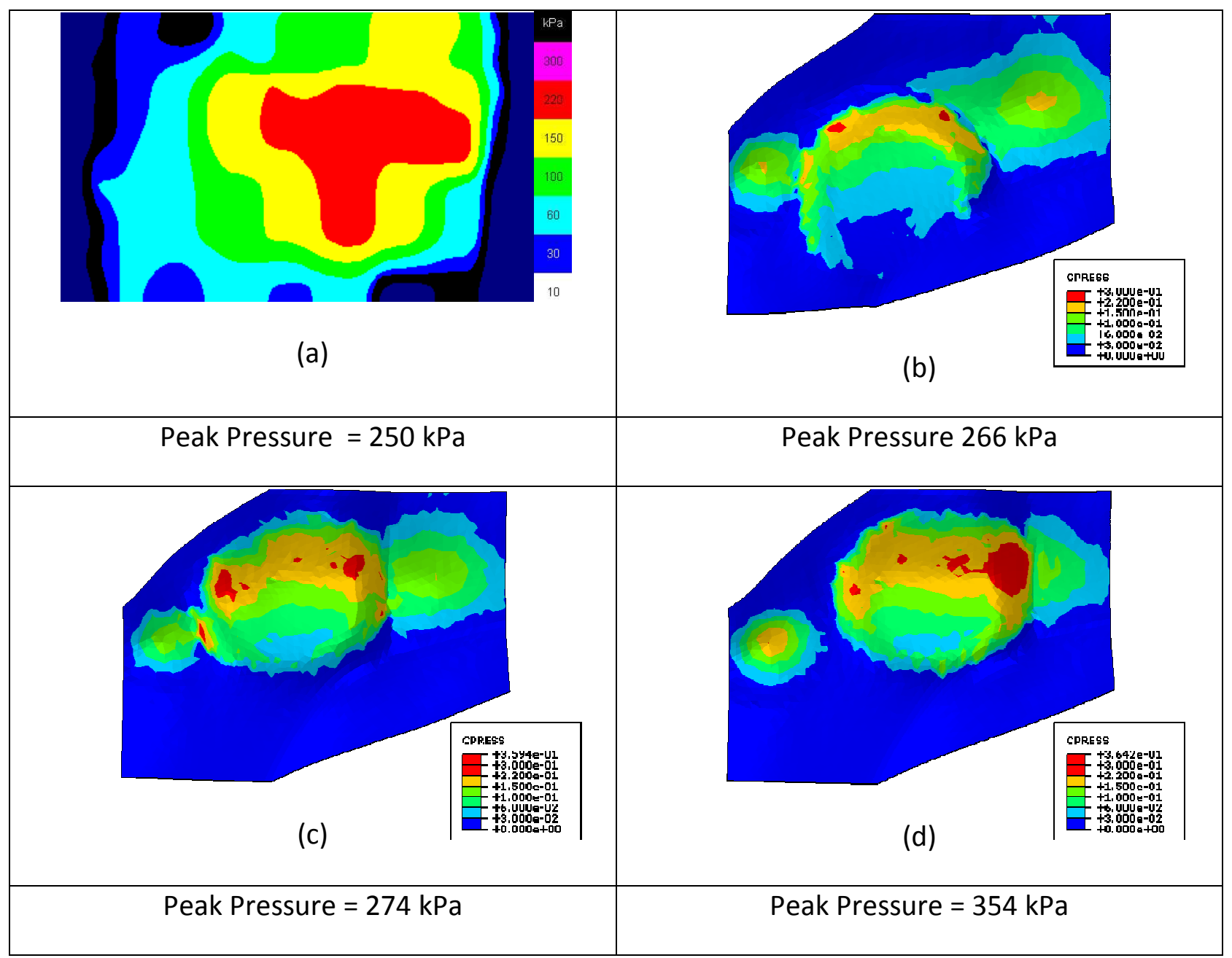




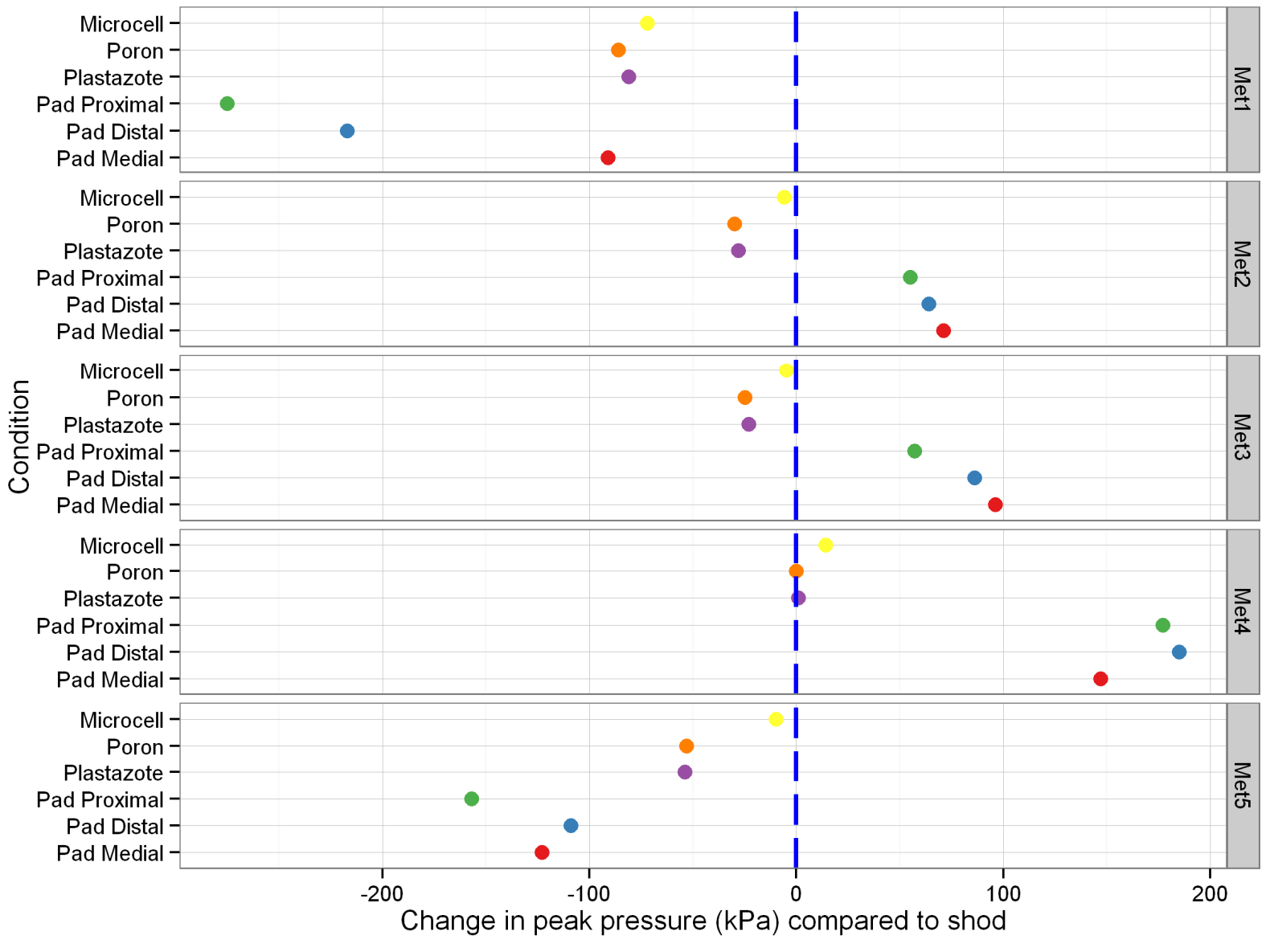




\section{References}

Abaqus 6.10. Abaqus 6.10 User Manual [Internet]. 2010 [cited 2010]. Available from: http://simulia.com

Actis R, Ventura L, Lott D, Smith K, Commean P, Hastings M et al. Multi-plug insole design to reduce peak plantar pressure on the diabetic foot during walking. Medical and Biological Engineering and Computing. 2008;46(4):363-71.

Boulton A, Kirsner R, Vileikyte L. Neuropathic diabetic foot ulcers. New England Journal of Medicine. 2004;351(1):48-55.

Cheung J, Zhang M. Parametric design of pressure-relieving foot orthosis using statistics-based finite element method. Medical engineering \& Physics. 2008; 30(3):269-77.

Cheung JTM, Zhang M. A 3-dimensional finite element model of the human foot and ankle for insole design. Arch Phys Med Rehabil. 2005;86(2):353-8.

Erdemir A, Viveiros ML, Ulbrecht JS, Cavanagh PR. An inverse finite-element model of heel-pad indentation. J Biomech. 2006;39(7):1279-86.

Franciosa P, Gerbino S, Lanzotti A, Silvestri L. Improving comfort of shoe sole through experiments based on CAD-FEM modeling. Med Eng Phys. 2012;

Hennessy K, Woodburn J, Steultjens MPM. Custom foot orthoses for rheumatoid arthritis: A systematic review. Arthritis care \& research. 2012;64(3):311-20.

Hsu Y, Gung Y, Shih S, Feng C, Wei S, Yu C et al. Using an optimization approach to design an insole for lowering plantar fascia stress-a finite element study. Ann Biomed Eng.

2008;36(8):1345-52.

Isvilanonda V, Dengler E, laquinto JM, Sangeorzan BJ, Ledoux WR. Finite element analysis of the foot: model validation and comparison between two common treatments of the clawed hallux deformity. Clin Biomech (Bristol, Avon). 2012 Oct;27(8):837-44. 
Morag E, Pammer S, Boulton A, Young M, Deffner K, Cavanagh P. Structural and functional aspects of the diabetic foot. "Clin Biomech (Bristol. 1997;12(3):S9-10.

Petre M, Erdemir A, Cavanagh P. Determination of elastomeric foam parameters for simulations of complex loading. Comput Methods Biomech Biomed Engin. 2006;9(4):231-42.

Petre M, Erdemir A, Panoskaltsis VP, Spirka TA, Cavanagh PR. Optimization of nonlinear hyperelastic coefficients for foot tissues using a magnetic resonance imaging deformation experiment. J Biomech Eng. 2013;135(6):61001-12.

Qiu TX, Teo EC, Yan YB, Lei W. Finite element modeling of a 3D coupled foot-boot model. Med Eng Phys. 2011;33(10):1228-33.

Tadepalli SC, Erdemir A, Cavanagh PR. Comparison of hexahedral and tetrahedral elements in finite element analysis of the foot and footwear. J Biomech. 2011;44(12):2337-43.

Waaijman R, Arts MLJ, Haspels R, Busch-Westbroek TE, Nollet F, Bus SA. Pressure-reduction and preservation in custom-made footwear of patients with diabetes and a history of plantar ulceration. Diabet Med. 2012.

Yarnitzky G, Yizhar Z, Gefen A. Real-time subject-specific monitoring of internal deformations and stresses in the soft tissues of the foot: a new approach in gait analysis. J Biomech. 2006;39(14):2673-89. 